\title{
Removing Shadows from Video
}

\author{
Seyed Mahdi Javadi Brunel, Yongmin Li, and Xiaohui Liu
}

\begin{abstract}
This paper presents a novel approach to automatic shadow identification and removal from video input. Based on the observation that the length and position of a shadow changes linearly over a relatively long period in outdoor environments, due to the relative movement of the sun, we can distinguish a shadow from other dark regions in an input video. Subsequently, we can identify the Reference Shadow as that with the highest confidence of the aforementioned linear changes. This Reference Shadow is used to fit the shadow-free invariant model, with which the shadow-free invariant images can be computed for all frames in the input video. Our method does not require camera calibration and shadows from stationary objects, as moving objects are detected automatically.
\end{abstract}

Index Terms-Invariant image, reference shadow, video surveillance, shadow-less image, shadow detection.

\section{INTRODUCTION}

Identifying shadows in images and videos is a significant issue in Image Processing, with potentials in numerous application; for example, object recognition, tracking, and video surveillance. To detect and track an object correctly, such as a car or a pedestrian, one frequently needs to identify the shadows in images and videos. When an object enters a shadow region, tracking may fail or its performance may be adversely affected. Moreover, shadows of moving objects can be identified mistakenly as separate items or a component of the same object. This paper introduces a new method that can be used to detect and remove shadows in videos. This method is developed to address a specific problem: shadow detection from stationary cameras in the outdoor environment.

Our method is inspired simply by the observation that the position and size of the shadow cast by a stationary object changes approximately linearly, consequent to the relative movement between the sun and the object. A dark region in an image can be either a shadow or a dark object. However, if we record the positions of several dark regions from consecutive frames of a video input over a long period, some shadows, though not all, can be identified relatively easily. This is because the positions of these shadows change linearly, while those of the dark object do not. It should be noted that our method requires the detection of only one shadow with the highest confidence. Once this is achieved, we can use it as a Reference Shadow to estimate the parameters necessary to generate a grey-scale invariant image [1].

The other advantage of our method is camera calibration

Manuscript received July 20, 2017; revised October 13, 2017.

The authors are with the Department of computer science, Brunel University, UK (e-mail: Seyed.javadi@brunel.ac.uk, Yongmin.li@brunel.ac.uk, XiaoHui.Liu@brunel.ac.uk). is not required; moreover, shadows from moving and stationary objects will be detected upon identification of the Reference Shadow.

The remainder of the paper is organised as follows. Section II provides the background to this topic while Section III explains the Reference Shadow and how it can be identified. Section IV presents a new method of detecting the remaining shadows in the scenes once the Reference Shadow is identified. Section V outlines our experiments and Section VI provides a summary of this paper.

\section{BACKGROUND}

Significant development has been made in the area of shadow detection and removal. In one of Barrow and Tenenbaum's early works [2], the concept of intrinsic images was introduced. Intrinsic images are a mid-level transform of the observed images. They are viewpoint-dependent and the physical causes of changes in illumination at different points are not made explicit. Barrow and Tenenbaum explained that this mid-level transform can be very useful for supporting a range of visual inferences. Multiple frames have been used to compute the intrinsic images [3]. They approached the subject of shadow detection is by formulating this problem as a maximum-likelihood (ML) estimation problem based on the assumption that derivative-like filter outputs applied to illumination have a tendency to be sparse. They derived the ML estimator under this assumption and demonstrated its suitability for recovering reflectance. Furthermore, they assumed that filter outputs are independent across space and time. In this method, Weiss used 35 images from morning until evening, with which our results are compared. Our method has the advantage of identifying shadows in a similar noisy environment in approximately 10 minutes. [4] and [5] used multiple frames, but it is not clear how these methods will perform when the scene contains other changes, such as multiple object movement. Moreover, this method can be useful in a controlled environment, but the impact of input noise is unknown. There are other methods that detect shadows based on the previous positions and the use of the sun [6], or concerning calibration methods using the sun, and the sky [7] or the shadows position [8]. These methods require significant training data and need additional information pertaining to the scene such as GPS location of the camera, date and time of the day. However, none of this information is required in our method.

In recent years, other methods have been developed to remove the effect of illumination from a single image [9]-[12]. These methods are based primarily on distinguishing between the texture of the objects and shadows. Bell and Freeman [9] took a learning base approach and generated a training set of images containing 
shading and reflectance variations. In [10], initially, a part labelling that densely covers the object is defined. Next, the Layout Consistent Random Field (LayoutCRF) model imposes asymmetric local spatial constraints on labels to ensure the consistent layout of parts. Tappen, Freeman and Adelson [11] have used multiple cues to recover shading and reluctance intrinsic images from a single image. They used both colour information and a classifier which is trained to recognise greyscale patterns in images; each image is then classified as being caused by shading or a change in the reluctance.

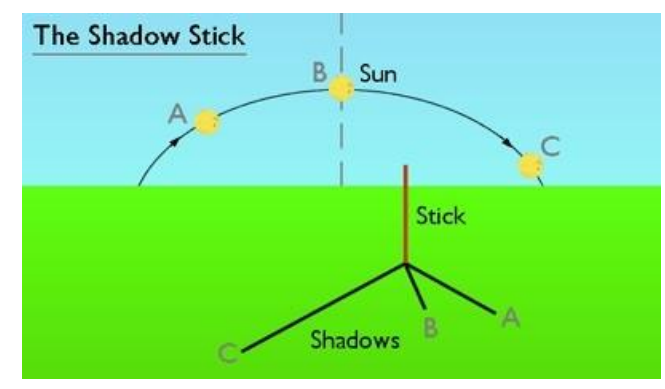

$L$

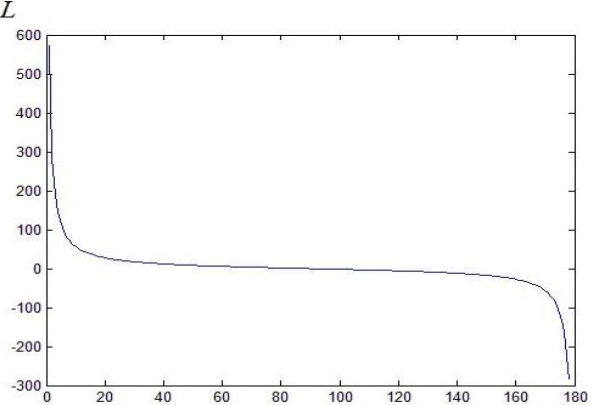

Fig. 1. (a) The shadow of a stick moves from $A$ to $B$ and then $C$ during the day, when the sun goes from A to B and then C. (b) Length of reference shadow from sunrise until sunset. The pattern is linear during most of the day; apart from sunrise and sunset.

Additionally, semi-supervised learning based methods have been developed to detect shadows. These methods require at least one shadow to be labelled by a user before identifying the remaining shadows [13]-[15]. The Neural Network is another popular method for detecting shadows, typically requiring a large number of labelled training images [16]-[18]. Faghih and Moghaddam [19] used image statistics to model the accuracy of Grey-Edge assumption in order to compensate for the Grey-Edge algorithm error. They used Weibull distribution to describe image statistics based on image derivatives. Cavallaro, Salvador and Ebrahimi [20] have tried to detect shadows from a sequence of images by processing three sources of information; namely, colour, spatial, and temporal information for the scene. Foreground objects were first segmented from the background, before successfully detecting shadows from the foreground objects. The performance of the aforementioned methods varies when dealing with changing lighting conditions. Typically, these methods require additional manual parameter adjustments in order to adapt it to different environments. To solve this issue, various studies use Gaussian to model shadows dynamically; However, good results are achieved only when the scene meets a series of assumptions [21]-[23]. Another important study on this topic is published by Finlayson et al. [1], who introduced the concept of greyscale invariant image and presented a computational model to estimate the invariant image. Same authors published another study and created invariant image by Entropy Minimization [24]. Our research is inspired by the work of Finlayson et al., but we used Reference Shadows instead of camera calibration to automatically create invariant images.

\section{REFERENCE SHADOWS}

This paper proposes a new method of detecting daytime outdoor shadows. Due to the relative movement between the sun and an object, the position and length of the shadow cast by the object changes throughout the day. Here, we investigate how this information can be used in real-life scenarios to assist in detecting shadows.

As illustrated in Fig. 1, the length of a shadow $L$ can be computed as

$$
L=h / \tan (\alpha)
$$

where $h$ is height of the object casting the shadow and $\alpha$ is the angle between sun and the horizon. The value of $\mathrm{L}$ for $\alpha$ between 0 to 180 degrees is presented in Fig. 2. This demonstrates that when the sun rises and moves from east to west, the length of the shadow moves in a pattern that is very close to a line apart. The exception occurs in early morning and just after sunrise and before the sunset when a non-linear pattern is observed. We analyse and process the position of the centre of the shadow instead of its length. In a short period, the changes to the position of the centre of the shadow will have a similar pattern to the length.

Our experiments confirmed monitoring the centre of the shadow will be sufficient to distinguish Reference Shadow from other dark objects. Additionally, the impact of other factors, such as object shape and camera view, will be minimal in short sampling periods, and will not alter the trajectory of the centre (or corner) of the shadow. Based on our experiments, using the corner of shadows will improve the performance of shadow detection system when the shadows are located at image boundaries. The Reference Shadow is the key element in detecting the shadows in the image and it is determined as: a dark part of the image which can be categorised as shadow with the highest confidence. Once we have identified at least one Reference Shadow, the remaining shadows (from stationary and moving objects) can be detected, as described in the next section.

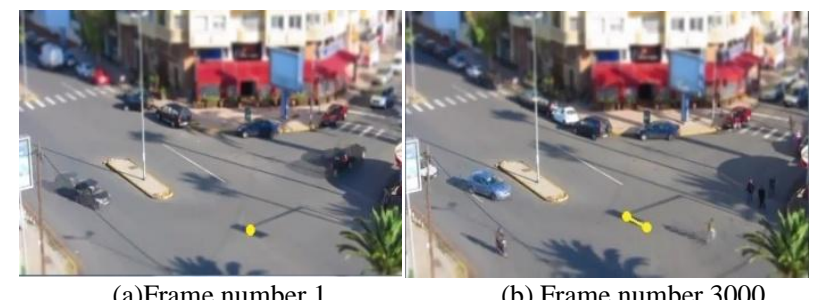

(a)Frame number 1 .

(b) Frame number 3000

Fig. 2. The centre of the shadow (marked in yellow) has changed in 3000 frames.

\section{A. Pre-processing}

As explained above, the height of the sun varies during the day; hence, the length and position of the shadows 
change. This can be used as an excellent cue for detecting some of the shadows. To do this, we must monitor some aspects (such as centre or corner) of dark objects over a period of time. In most real-time scenarios, the result will be very noisy because objects move over the shadows and there might be some minor camera movement etc. Nevertheless, the patterns of shadows and dark regions will differ entirely over time. To explain this in more detail, we will use the example given in Fig. 3 which is based on 3000 frames taken from a 20-minutes surveillance camera video. The camera is directed at a busy road junction in Morocco.

In the above example, the following steps have been taken to detect a Reference Shadow:

1) The RGB image is converted to binary image.

2) The binary image is filtered using an averaging filter.

3) The position of the centre of the black regions is recorded across all frames following the registration process.

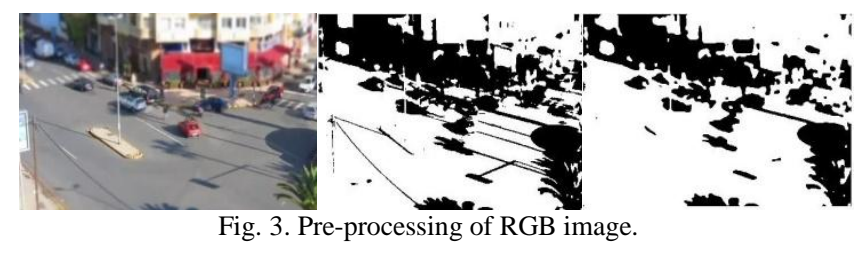

This process is illustrated in Fig. 3.

\section{B. Registration Process}

Now that Fig. 4 (c) is produced, we introduced a registration process to track and analyse the position of the centre of dark regions across all available frames. This also ensures the categorisation of dark regions have been completed with minimum error. To achieve this, we register dark regions in each frame only if both of the following two conditions are met:

1) The position of the centre of the dark region in the new frame should be very close to that in the previous registered frame.

2) The size (number of pixels) of the dark region in the new frame should be very close to that in the previous registered frame.

If any of the above conditions is not met, we discard the dark region and do not register the coordination of the centre. Instead, we select 0 for the value of $\mathrm{x}$ and $\mathrm{y}$ of the frame's dark region.

In addition, we have used a counter to determine the confidence in our readings. When there is a new reading, the counter will be increased by one; hence, at each point in time, we know the confidence in each dark region by comparing the value of the counter of each dark region.

\section{Selection Process}

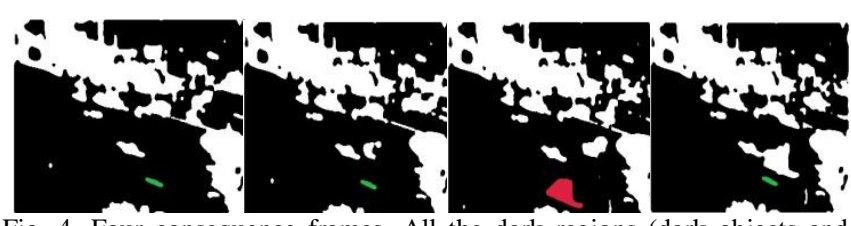

Fig. 4. Four consequence frames. All the dark regions (dark objects and shadows) are highlighted in white. The registered dark regions are displayed in green and the unregistered dark region is displayed in red. The reason the dark region in frame (c) is not registered is because another object (possibly a car) has gone over the dark region and hence the size of this dark region has changed significantly from previously registered frame.
When all the frames have completed the pre-processing and registration process, we recorded the positions of the centre all of the registered dark regions and stored this data in a matrix. The results are displayed in Fig. 4.

In Fig. 5(a) and Fig. 5(b), the $x$ values of the centre of two dark regions are displayed. Region A is one shadow (or part of the shadow) of a stationary object; possibly a road sign, and region $\mathrm{B}$ is the dark car which is parked and not moving during the 20-minute recording. These two regions are marked in Fig. 5(a). The gaps in the readings are due to the frames dropped during registrations process, as explained previously.

As illustrated in Fig. 5, the centre of the shadow region changes gradually while the centre of the dark object remains the same.

So, the key question to answer when detecting shadows is how we can distinguish between dark objects and shadows; the answer is given in Fig. 5 (a) and Fig. 5 (b). If we record the dark regions for a relatively long period and then check the trajectory of the centre (or corners) of dark objects, the shadow and non-shadow regions will reveal different patterns.

This is precisely what we observed in the experiment from the surveillance video in Morocco. As illustrated in Fig. 5 (a), the centre of the shadow region changes linearly during the 20-minute observation at midday; thereby matching the pattern presented in Fig. 2. To implement this, we fit a line using the registered centres before checking its angle. If the angle of the line is horizontal, the centre has not changed over the time and the dark region is not a shadow. If the angle of the line is not 0 , we have successfully identified a Reference Shadow.

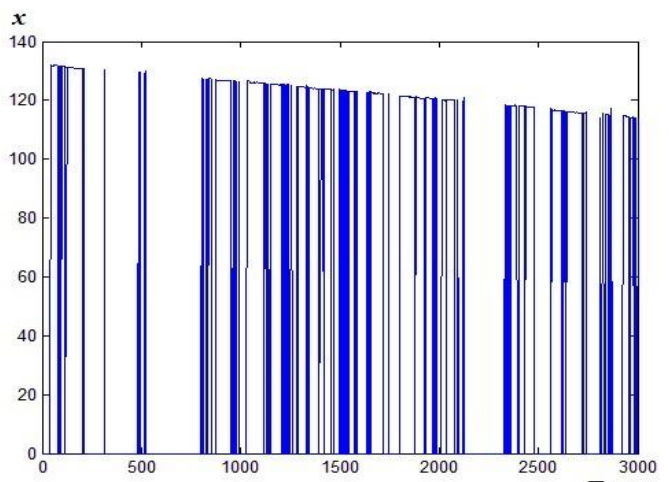

(a)

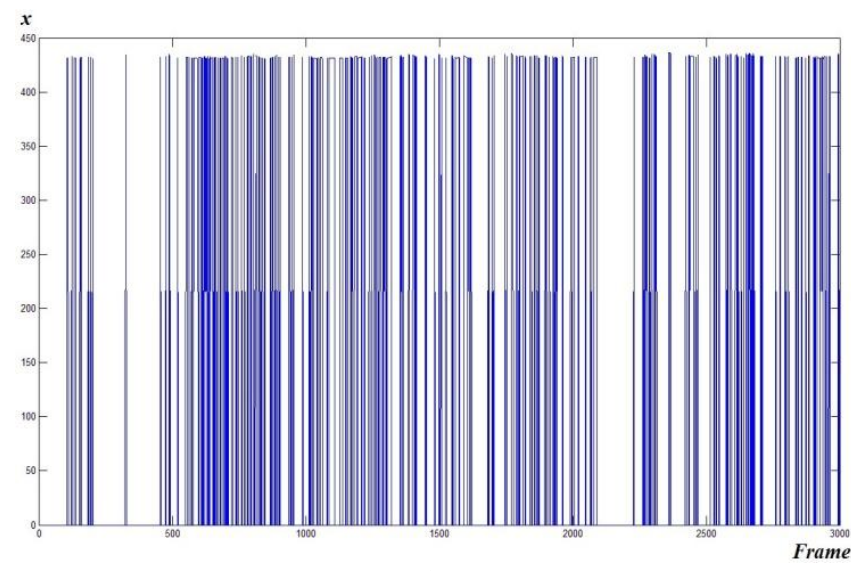

(b)

Fig. 5. (a) $x$ value of the centre of shadow over the 3000 frames and (b) $x$ value of the centre of dark over the 3000 frames. 


\section{Detecting The REMAINING SHADOWS}

Now that we have identified one Reference Shadow with high confidence, it is possible to detect the remaining shadows in the image. Our solution is based on identifying a grey-scale invariant image [1], which is defined as:

$$
g s=c_{1} R^{\prime}(R)-c_{2} R^{\prime}(B)
$$

where in RGB space, $R^{\prime}(R)=\log (R / G)$ and $R^{\prime}(B)=$ $\log (B / G) . c_{1}$ and $c_{2}$ are constants such that the vector $\left[c_{1} c_{2}\right]$ is in the direction is orthogonal to the lighting direction.

Experiments have confirmed images with different levels of illumination will map to the same grey-scale invariant image. The most important benefit of this image is shadows (which occur when there is a change in luminance) will disappear. Now, if we divide (2) by $c_{1}$, we get the following:

$$
\left.g s=R^{\prime}(R)-C R^{\prime} B\right)
$$

where $C=c_{2} / c_{1}$ Others, such as Finlayson [1], have used manual camera calibration to identify $c_{1}$ and $c_{2}$ but we use the Reference Shadow to automatically create a grey-scale invariant image by estimating the value of $C$. To create the invariant image without camera calibration, we generate a large number of $g s^{0}$ with different values of $C$ in $[0,1]$. At some point, $\left[c_{1}, c_{2}\right]$ becomes orthogonal to lighting direction and shadow edges will disappear. The following steps are taken to automatically detect the shadow edges:

\footnotetext{
Algorithm 1 Generating grey-scale invariant image

1: Select $C \in[0,1]$ to generate $g s^{0}$ samples.

2: For all $g s^{0}$ do

3: Create Edge Map. (we used Sobel method).

4: Take multiple block samples from inside and outside of Reference Shadows boundary.

5: Find the gs sample with the least difference between inside and outside the edge.

6: End For

7: The gs sample identified in step 5 is grey-scale invariant image and the corresponding parameter $C$ is set.
}

The following example which is illustrated in Fig. 6 is from a video taken from a stationary camera. We have used one frame per 2.5 seconds and a total of 3000 frames were used to detect the Reference Shadow. In this case, we correctly detected the shadow located at the centre of the image as the Reference Shadow and used it to generate the grey-scale invariant image.

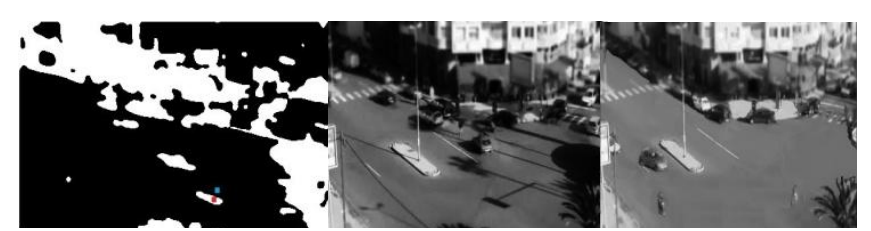

Fig. 6. After processing 3000 frames, the shadow at the centre of the image is identified as a Reference Shadow. (a) Comparing the values of a pixel block across the edge of Reference Shadow. (b) One of the gs samples where the Reference Shadow is not removed. In this image $\mathrm{C}=0.71$ (c) One of the gs samples with the least difference between inside and outside Reference Shadow. In this sample, $\mathrm{C}=0.42$.

\section{EXPERIMENT}

The experiments outlined in Fig. 7 are based on videos taken from a stationary camera. They reveal how the position of the centre of the Reference Shadow changes as time passes. The goal of these experiments is to demonstrate the possibility of detecting at least one Reference Shadow in most real-life scenarios. Once the Reference Shadow is detected, the grey-scale invariant image can be computed for the whole video. We have compared our method with the work of Weiss [2], Matsushita [4] and other state-of-art methods on the removal of shadows from videos. The reason we selected Wiess and Matsushita's methods for comparison is both methods were designed for outdoor shadow removal from video and require multiple samples for model learning. Overall, our method is very robust in many complicated cases such as noisy environment with moving objects.
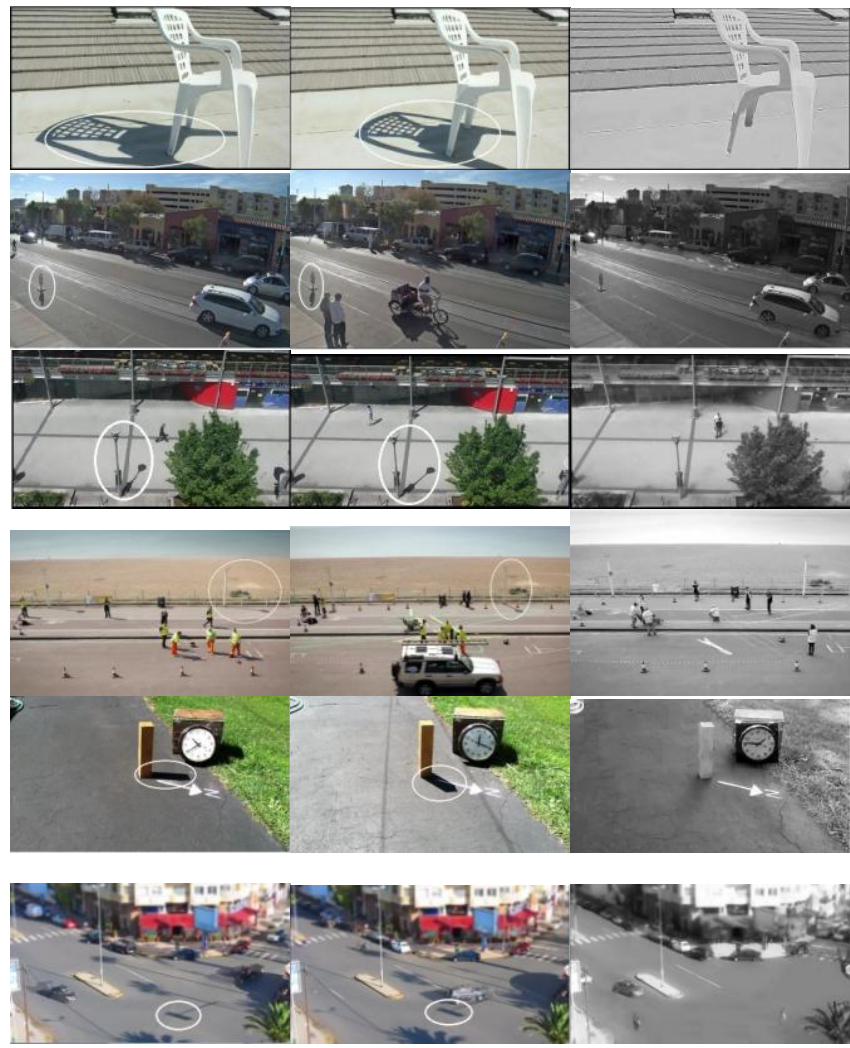

Fig. 7. Detecting Reference Shadows in various environments. The first two columns are frames that display the position of Reference Shadow, while the last is the grey-scale Invariant Image.

\section{COMPARISON WITH OTHER METHODS}

We have compared our method with the work of Weiss [3], Matsushita [5] and other state-of-the-art methods of shadow removal from videos. The reason we selected Wiess and Matsushita's methods for comparison is both methods were designed for outdoor shadow removal from video, and require multiple samples for model learning.
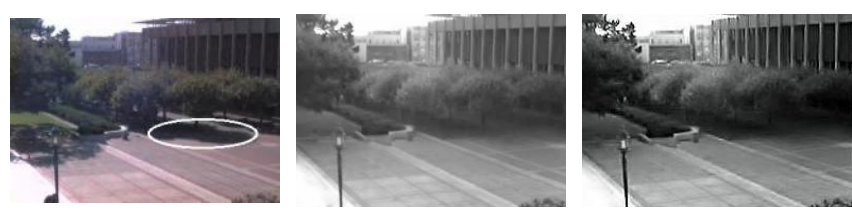

Fig. 8. (a) is an original imput image. (b) is shadow-less image based on Weiss and (c) is based on Reference Shadow. The frame samples used in [3] are from morning until sunset. However, video footage for around 15 minutes will be sufficient to detect Reference Shadow using our method. The final grey-scale results are very similar; however, the advantage of our method is it will detect shadows a lot faster. 
Overall, in outdoor scenarios, our method is very robust in many complicated cases such as noisy environment with moving objects as well as presence of moving clouds. However, what makes our method stand out is its simplicity and the time required to successfully detect a Reference Shadow. The majority of other video-based methods require hours of training data or manual intervention (such as camera calibration). Nonetheless, we managed to detect shadows in as little as 10 minutes in extremely complex noisy environments containing numerous moving objects.

Table I highlights the advantages of our method over some of the existing methods in the outdoor domain. To demonstrate how our system performs, we applied our method to the image set established by Weiss [3] and Matsushita [5]. In terms of quality of the shadow-less images, there is no noticeable difference. Moreover, both methods yield very similar results, as presented in Fig. 8 and Fig. 9.

\begin{tabular}{|cccc|}
\hline \multicolumn{1}{|c}{ TABLE I: OUTDOOR SHADOW DETECTION METHODS } & $\begin{array}{c}\text { Initialisation } \\
\text { Period }\end{array}$ \\
\hline Refhod & $\begin{array}{c}\text { Indoor/Outdoor } \\
\text { Shadows }\end{array}$ & $\begin{array}{c}\text { Background/Foreground } \\
\text { Shadows }\end{array}$ & $\begin{array}{c}\text { Mequires GPS } \\
\text { Location }\end{array}$ \\
Weiss & Outdoor & Both & No frames in one day \\
Matsushita & Both & Both & Hours \\
Finlayson & Outdoor & Both & Requires camera calibration \\
Cavallaro & Both & Both & Requires background subtraction \\
Huerta & Both & Only Foreground & Single Image \\
Antone & Outdoor & Only Foreground & Hours \\
El-Zahhar & Outdoor & Both & Semi-supervised \\
Boroujeni & Both & Both & Hours
\end{tabular}

However, a significant difference exists between the two methods in terms of the time required to produce the invariant image. For example, Weiss used 35 frames from sunrise to sunset, but in our experience, footage of approximately 10 minutes will be sufficient to detect shadows. The same results were observed when comparing our method with Matsushita's method [5]. Hence, our method is a very strong solution for real-time application, as it can detect shadows in a few minutes.
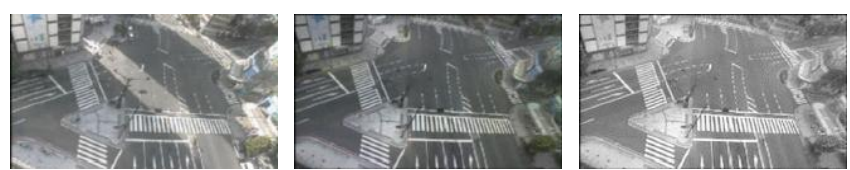

Fig. 9. (a) is an original image Matsushita [5]. (b) is shadow-less image based on Matsushita and (c) is based on Reference Shadow. They used multiple in [5] are from different time of the day. However, a short video footage from similar scenario was used to detect Reference Shadow using our method. the advantage of our method is it detect shadows significantly quicker and can be used when there are moving objects. Matsushita's method produces colour shadow-less image, but assumes there are no moving objects in the scene.

\section{CONCLUSION AND FURTHER WORK}

This paper has introduced a new method to automatically remove shadows in videos taken from stationary cameras in an outdoor environment. Based on the observation that, the length and position of a shadow changes linearly over a relatively long period of time in a outdoor environment, due to the relative movement of the sun, we can distinguish a shadow from other dark regions in an input video. Subsequently, we can identify the Reference Shadow as having the highest confidence of the aforementioned linear changes. This Reference Shadow is used to fit the Finlayson shadow-free invariant model [1], with which the shadow-free invariant images can be computed for all frames in the input video.

The main contributions of the work can be summarised as follows:
1) We have developed a novel method of identifying the Reference Shadow. Without any prior knowledge, a shadow would appear the same as other dark regions in an image; for example, an object with dark colours. However, if we observe the changing patterns of the position and length of a dark region, those for a shadow changes linearly while those for a dark object remain the same. Thus, the shadows can be distinguished from the dark objects. With simple confidence measured against this linearity and consistency of changes, we can select one or a few Reference Shadows with the highest confidence. Experimental results have demonstrated this process of Reference Shadow identification is fairly accurate and reliable.

2) With the Reference Shadows, we can effectively fit the Finlayson shadow-free invariant model [1], by simply computing the accumulated profiles across the shadow boundaries. Previously, this model fitting normally involved troublesome camera calibration, but with our new method, the process becomes straightforward and automatic.

\section{ACKNOWLEDGMENT}

The authors would like to thank Professor Yair Weiss for sharing his experiment data.

\section{REFERENCES}

[1] G. Finlayson, S. Hordley, and M. Drew, "On the removal of shadows from images," IEEE Transactions on Pattern Analysis and Machine Intelligence, vol. 28, no. 1, pp. 59-68, 2006.

[2] H. Barrow and J. Tenenbaum, "Recovering intrinsic scene characteristics from images," Computer Vision systems, vol. 1, no. 23, pp. 3-26, 1978.

[3] Y. Weiss, "Deriving intrinsic images from image sequences," in Proc. IEEE International Conference on Computer Vision, Vancouver, Canda, Jul. 2001, pp. 68-75.

[4] Y. Matsushita, S. Lin, S. Kang, and H. Shum, "Estimating intrinsic images from image sequences with biased illumination," in Proc. European Conference on Computer Vision, Prague, Czech Republic, May 2012, pp. 274-276. 
[5] Y. Matsushita, K. Nishino, K. Ikeuchi, and M. Sakauchi, "Illumination normalization with time-dependent intrinsic images for video surveillancel," vol. 26, no. 10, pp. 1336-1341, 2004.

[6] I. Huerta, M. Holte, and T. Moeslund, "Detection and removal of chromatic moving shadows in surveillance scenarios," in Proc. IEEE Conference on Image Processing, Kyoto, Japan, Sep. 2009, pp. 1499-1506.

[7] J. Lalonde, S. Narasimhan, and A. Efros, "What do the sun and the sky tell us about the camera?" in Proc. International Conference on Computer vision, Berlin, Germany, Sep. 2008, pp. 354-367.

[8] M. Antone and M. Bosse, "Calibration of outdoor cameras from cast shadows," in Proc. IEEE Conference on Systems, Man and Cybernetics, Burlington, USA, 2014.

[9] M. Bell and W. Freeman, "Learning local evidence for shading and reflection," in Proc. International Conference in Computer Science, vol. 1, 2001, pp. 670-675.

[10] J. Winn and J. Shotton, "The layout consistent random field for recognizing and segmenting partially occluded objects," in Proc. IEEE Computer Society Conference on Computer Vision and Pattern Recognition, NYC, USA, Jun. 2006, pp. 37-44.

[11] M. Tappen, W. Freeman, and E. Adelson, "Recovering intrinsic images from a single image," IEEE Transactions on Pattern Analysis and Machine Intelligence, vol. 27, issue 9, pp. 1459-1472, 2005.

[12] L. Shen, P. Tan, and S. Lin, "Intrinsic image decomposition with non-local texture cues," in Proc. IEEE Conference on Computer Vision and Pattern Recognition, Anchorage, USA, Jun. 2008, pp. $1-7$.

[13] M. El-Zahhar, A. Karali, and M. ElHelw, "A semi supervised learning-based method for adaptive shadow detection," in Proc. IEEE Confrance on Signal and Image Processing Applications, Kuala Lumpur, Malaysia, Nov. 2012, pp. 384-353.

[14] H. Boroujeni, N. Charkari, and A. Jalilvand, "A robust moving shadow detection algorithm based on semisupervised hierarchical mixture of mlp-experts," in Proc. International Conference on Soft Computing and Pattern Recognition, Dalian, China, Oct. 2011, pp. 141-146.

[15] J. Zhao, S. Kong, and G. Men, "A robust moving shadow detection algorithm based on semi-supervised hierarchical mixture of mlp-experts," in Proc. Chinese Control and Decision Conference, Mianyang, China, May 2011, pp. 1672-1676.

[16] V. Agarwal, A. V. Gribok, and M. A. Abidi, "Machine learning approach to color constancy," Neural Networks, vol. 14, no. 3, pp. 559-563, 2007.

[17] V. Cardei, B. Funt, and K. Barnardi, "Estimating the scene illumination chromaticity using a neural network," Optical Society of America, vol. 9, no. 1, pp. 2374-2386, 2002.

[18] R. Stanikunas, H. Vaitkevicius, and J. Kulikowski, "Estimating the scene illumination chromaticity using a neural network," Neural Networks, vol. 17, no. 3, pp. 327-337, 2004.

[19] M. Faghih and M. Moghaddam, "Neural gray edge: improving gray edge algorithm using neural networks," in Proc. IEEE Conference on Image Processing, Brussels, Belgium, Jun. 2011, pp. 1705-1708.

[20] A. Cavallaro, E. Salvador, and T. Ebrahimi, "Detecting shadows in image sequences," in Proc. First European Conference on Visual Media Production, London, UK, Jun. 2004, pp. 15-16.
[21] J. Huang and C. Chen, "Moving cast shadow detection using physics-based features," in Proc. IEEE Conference on Computer Vision and Pattern Recognition, Miami, USA, Jun. 2009, pp. 2310-2317.

[22] A. Jalilvand and N. Charkari, "A novel coloured local invariant descriptor based on surf," in Proc. International Conference on Computer and Knowledge Engineering, Oct. 2011, pp. 214-219.

[23] F. Porikli and J. Thornton, "Shadow flow: a recursive method to learn moving cast shadows," in Proc. IEEE Conference on Computer Vision, Miami, USA, Oct. 2005, pp. 891-898.

[24] G. D. Finlayson, M. S. Drew, and C. Lu, "Entropy minimization for shadow removal," International Journal of Computer Vision, Miami, USA, May, pp. 35-57, 2009.

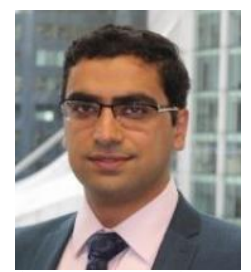

Seyed Mahdi Javadi Brunel received B.Eng degree in computer science from Shahid Behesht University University, Tehran, Iran, in 2003 and MSc in data communication systems from University of Brunel, UK, in 2006 respectively, he has been holding senior roles in IT industry in UK since 2006 and he is currently a $\mathrm{PhD}$ student at University of Brunel. His research interests are image processing, computer vision, tracking and video surveillance.

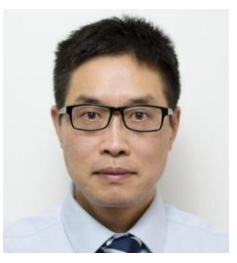

Yongmin Li received the M.Eng. and B.Eng. degrees in control engineering from Tsinghua University, Beijing, China, in 1990 and 1992 respectively, and the $\mathrm{Ph} . \mathrm{D}$. degree in computer vision from Queen Mary University of London, London, U.K., in 2001.Between 2001 and 2003, he worked as a Research Scientist at the British Telecom Laboratories, Suffolk, U.K. He is currently a senior lecturer in the Department of Computer Science, Brunel University, West London, U.K. His current research interests include automatic control, nonlinear filtering, computer vision, image processing, video analysis, medical imaging, machine learning, and pattern recognition.

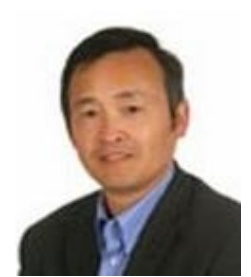

Xiaohui Liu is professor of Computing and Director of the Centre for Intelligent Data Analysis at Brunel University London. He is interested in taking an interdisciplinary approach to meeting the challenges in integrating, analysing and visualising a variety of high-dimensional, fast evolving, and complex data, especially in the modern era of big data. Professor Liu is a chartered engineer, chartered fellow of the British Computer Society, fellow of the Royal Statistical Society, and life member of the Association for the Advancement of Artificial Intelligence. 